Research Article

\title{
Scale production of conductive cotton yarns by sizing process and its conductive mechanism
}

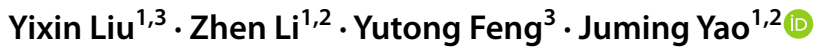

Received: 13 October 2020 / Accepted: 9 March 2021

Published online: 10 May 2021

(c) The Author(s) 2021

OPEN

\begin{abstract}
Conductive yarn is an important component and connector of electronic and intelligent textiles, and with the development of high-performance and low-cost conductive yarns, it has attracted more attention. Herein, a simple, scalable sizing process was introduced to prepare the graphene-coated conductive cotton yarns. The electron conductive mechanism of fibers and yarns were studied by the percolation and binomial distribution theory, respectively. The conductive paths are formed due to the conductive fibers' contact with each other, and the results revealed that the connection probability of the fibers in the yarn $(p)$ is proportional to the square of the fibers filling coefficient $(\varphi)$ as $p \propto \varphi^{2}$. The calculation formula of the staple spun yarn resistance can be derived from this conclusion and verified by experiments, which further proves the feasibility of produce conductive cotton yarns by sizing process.
\end{abstract}

Keywords Conductive cotton yarns · Sizing · Filling coefficients · Percolation threshold · Binomial distribution

\section{Introduction}

With the development of wearable electronic devices, traditional textiles have gradually been upgraded to intelligent and electronic textiles, and its application prospects in sensors, supercapacitors, connectors, battery energy convertors, energy harvesting, and storage devices were also proved and described by many publications $[2,12,13$, $15,22,23,27,29]$. The vital problem of electronic textiles was to adopt flexible conductive wires instead of the rigid metal wires for electrical signal transmission, so the growing interests were focused on yarn-based conductive wires because the yarns were inherently soft and can process into the desired textiles $[16,30]$.

At present, two types of fabrication conductive yarns are widely accepted and adopted. One is directly processing intrinsically conductive continuous filaments (carbon or metal-based) placed alongside (plied) with nonconducting synthetic filaments or discontinuous cut staple fibers blended with nonconductive natural or synthetic fibers. Xu et al. [26] prepared the conductive yarns using graphene filaments which exhibited good electrical conductivity $\left(0.8 \times 10^{6} \mathrm{~S} \mathrm{~m}^{-1}\right)$. Asghar et al. [1] prepared the hybrid conductive yarns by twisting polyester and copper filaments which also exhibited good electrical

Supplementary Information The online version contains supplementary material available at https://doi.org/10.1007/s42452-02104493-9.

$\square$ Juming Yao, yaoj@zstu.edu.cn | ${ }^{1}$ The Key Laboratory of Advanced Textile Materials and Manufacturing Technology of Ministry of Education, College of Materials and Textiles, Zhejiang Sci-Tech University, 928 Second Avenue, Xiasha Higher Education Park, Hangzhou 310018, China. ${ }^{2}$ National Engineering Lab of Textile Fiber Materials and Processing Technology, Hangzhou 310018, China. ${ }^{3}$ College of Textiles and Clothing, Qingdao University, Qingdao 266071, China.

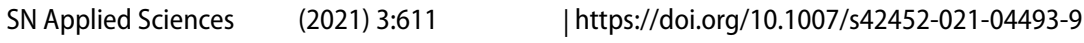


conductivity $(<0.023 \Omega \mathrm{cm})$. However, the relatively complicated fabrication (e.g., graphene filaments-based yarn) or the deterioration of flexibility and elastic recovery (e.g., metal filaments-based yarn) will hinder its application in wearable electronic textile. The other simple and more efficient method was using the existing yarns as substrates to integrate the conductive fillers into the conductive yarns by coating. Kim et al. [14] prepared conductive cotton yarns by dip-coating the reduce graphene oxide (RGO) on its surface which exhibited high resistance (3.21 M $\Omega$ ), and similarly Ma et al. [18] prepared conductive viscose yarn by dip-coating RGO on its surface with high resistance $(84.28 \mathrm{k} \Omega)$. Therefore, improving the long-term stability and washing fastness of yarns conductivity prepared by coating was still facing challenges. Our previous research demonstrated that the interaction between graphene and cellulose molecules is improved [17]. By simple dip-coating and hot press process, the resistance of the prepared cellulose/graphene composite flake (CGP) is $100 \Omega \mathrm{cm}$, which benefits from the good electrical conductivity of graphene. The sizing process commonly used in textiles includes impregnation and hot pressing. Inspired by this, we envisaged that the conductive yarn prepared by the sizing process improves yarn's conductivity and stability.

Herein, the sizing process was introduced to prepare conductive cotton yarns by using the graphene solution as size. The results indicate that the conductive mechanism of staple the spun yarn is related to the contact probability, and the connection probability was proportional to the fibers filling coefficients. A circuit model of the conductive yarn was established by analyzing the arrangement states of the spun yarn. Moreover, the resistance calculation formula of the spun yarn was further derived and verified by experiment. It is proved by experiment that the preparation of conductive spun yarn by the sizing process will expand the applications of cotton yarns.

\section{Experimental}

\subsection{Materials}

Graphene nanoplatelets (GnPs bulk density $0.15 \mathrm{~g} / \mathrm{cm}^{3}$ ) were kindly supplied by XG Science Inc, USA. Cotton yarns with fineness $7^{\mathrm{s}}(\approx 84 \mathrm{Tex})$ and $14^{\mathrm{s}}(\approx 42 \mathrm{Tex})$ were kindly supplied by Shenzhou textile Co., Ltd, China. Sodium hydroxide, urea, ethanol $(99.9 \% \mathrm{v} / \mathrm{v})$, and polyvinylpyrrolidone (PVP, $M_{n} 24,000$ ) were purchased from Shanghai Aladdin Chemical Reagent Co, Ltd, China. All the reagents were of analytical grade and used without purification.

\subsection{Preparation of GnPs solution and GnPs/ cellulose composite flakes}

The preparation of GnPs/cellulose solution contains three steps: the first step uses PVP/ethanol as a dispersing agent to prepare GnPs solutions; briefly, GnPs solutions were obtained by dispersing GnPs in ethanol with the aid of PVP (0.1-0.4 wt\%) under ultrasonication for 2-16 h. Secondly, cellulose solution was obtained by using $\mathrm{NaOH}$ /urea solutiondissolved cotton. At last, mix all solutions, ultrasonic, centrifugation, and filtration to remove the sediment. The actual content of GnPs in system was $0.76 \mathrm{mg} / \mathrm{mL}$ measured by gravimetric method, the progress of GNPs electronic slurry.

\subsection{Preparation of conductive cotton yarn by sizing}

The graphene solution with a concentration of $0.76 \mathrm{mg} / \mathrm{mL}$ as the size $(2000 \mathrm{~mL})$ and the conductive cotton yarns were fabricated by a single yarn sizing machine (SS600 CCI Tech. Inc.). Before sizing, cotton yarns were pretreated by alkalescent sodium hydroxide aqueous solution $(\mathrm{Ph}=8,2000 \mathrm{~mL}$ ) at the sizing process parameter of winding speed $20 \mathrm{~m} / \mathrm{min}$, sizing pressure $2 \mathrm{Mpa}$. Then, parameters were adjusted to winding speed $10 \mathrm{~m} / \mathrm{min}$, sizing pressure $5 \mathrm{Mpa}$ and hot drying temperature $150^{\circ} \mathrm{C}$ for $\mathrm{GnPs}$ solution sizing. The GnPs sizing process can be repeated some times to ensure that the sizing rate $\left(w \%=\frac{M_{j}-M_{0}}{M_{0}} \times 100 \%\right)$ above $2.0 \mathrm{wt} \% .\left(M_{j}, M_{0}\right.$ are the dry weight of cotton yarns of a certain length before and after sizing (Support Information S2 Video).

\subsection{Characterization of the properties of CGP and conductive cotton yarns}

The chemical structure of CGP was characterized by Fourier transform infrared spectroscopy (FTIR, Nicolet 5700, Thermo Electron Corp., USA) using $\mathrm{KBr}$ disk method in the range from 4000 to $400 \mathrm{~cm}^{-1}$ at a resolution of $4 \mathrm{~cm}^{-1}$. The crystal structure of CGP was characterized by X-ray diffraction diffractometer (XRD, D8 Advance, Bruker, USA) with a Cu Ka radiation $(\lambda=0.15405 \mathrm{~nm})$ with a scanning rate of $2^{\circ} \%$ $\mathrm{min}$ at $40 \mathrm{kV}$ and $40 \mathrm{~mA}$. The morphology of yarns after sizing were observed by scanning electron microscopy (SEM, 7900F, JEOL Japan).

\subsection{The porosity of CGP}

The porosity of CGP ( $\delta i)$ with different GnPs contents was measured by automatic true density and porosity analyzer (BSD-TDK Beshide Instrument Technology Co., Ltd, China) and is recorded in Table 1. The porosity of each samples was measured three times and averaged. 
Table 1 Parameters and electrical resistance of CGP with different graphene contents

\begin{tabular}{lccccccc}
\hline Sample no & CGP1 & CGP2 & CGP3 & CGP4 & CGP5 & CGP6 & CGP7 \\
\hline GnPs mass (g) & 0.0038 & 0.0076 & 0.0114 & 0.0114 & 0.0228 & 0.0304 & 0.038 \\
GnPs mass content (\%) & 0.35 & 0.70 & 0.105 & 0.105 & 2.10 & 2.80 & 3.50 \\
Density of CGP $\left(\mathrm{g} / \mathrm{cm}^{3}\right)$ & 0.98 & 1.02 & 1.05 & 1.05 & 1.08 & 1.09 & 1.09 \\
Porosity of CGP $(\%)$ & 36.4 & 33.5 & 32.2 & 32.2 & 28.4 & 27.0 & 26.3 \\
Packing density of GnPs $\left(\mathrm{g} / \mathrm{cm}^{3}\right)$ & 0.35 & 0.44 & 0.47 & 0.47 & 0.59 & 0.60 & 0.62 \\
Volume of GnPs $\left(\mathrm{cm}^{3}\right)$ & 0.011 & 0.017 & 0.024 & 0.024 & 0.039 & 0.507 & 0.062 \\
Volume fraction of GnPs(\%) & 1.57 & 2.40 & 3.36 & 3.36 & 5.35 & 6.79 & 8.24 \\
Conductivity of CGP $(\mathrm{S} / \mathrm{cm})$ & 0.013 & 0.035 & 0.105 & 0.105 & 0.532 & 0.883 & 1.660 \\
\hline
\end{tabular}

\subsection{The volume fraction and stacking density of GnPs in CGP}

The volume fraction and stacking density of GnPs in CGP were derived from the formula for calculating the porosity of composite materials and are recorded in Table 1 (Support Information S3).

\subsection{The electrical resistance of CGP}

The electrical resistance of CGP with different GnPs contents labeled CGP1-7 was measured by universal digital meter (Eyesight 34461 A, Agilent Technologies Inc., China) on ambient conditions $\left(20^{\circ} \mathrm{C}, 70 \mathrm{RH} \%\right)$. The conductivity of CGP can be calculated by equation $\sigma_{\text {com }}=\frac{L}{R A}(R, A, L$ is the resistance, cross sectional area and length of sample) and recorded in Table 1.

\subsection{The structural parameters of the conductive cotton yarns}

The main structural parameters of the conductive cotton yarns were measured after sizing and drying according to the general textile formula (Support Information S4), and all the parameters were obtained by the average of multiple measurements and are recorded in Table 2.

\subsection{The electrical resistance of conductive cotton yarn}

The electrical resistance of conductive cotton yarns was measured by universal digital meter on ambient conditions and is recorded in Table 3 as a control parameter comparison with the theoretically calculated value. The electrical resistance of the cotton yarns with length of $1 \mathrm{~m}$ under different temperature and humidity conditions was measured after drying and then keeping in constant temperature and humidity box for $4 \mathrm{~h}\left(20-120^{\circ} \mathrm{C}, 20-98\right.$ RH\%, YG751D, Wenzhou Dahua Textile Equipment Co., Ltd, China).

\subsection{Washing fastness of yarn resistance}

The washing fastness of yarn resistance was evaluated by the resistance change of the yarn with length of $1 \mathrm{~m}$ immersed in water for different times $(15,30,60,90,120$, $240 \mathrm{~min}$ ). The resistance of yarn was measured on ambient conditions after the yarn tanking out from water and drying.

\section{Results and discussion}

A staple spun yarn is a linear assembly of fibers, held together, usually by the insertion of twist, to form a continuous strand [7]. The conductivity of the staple spun yarn is based on the conductive properties of its contained fibers which can be effectively connected to form a complete conductive channel. Sizing is a necessary process for staple spun yarns before weaving, the purposes of which are to increase the adhesion of the fibers to improve the strength of the yarns by the penetration of the size into the yarns and to reduce hairiness by the size film formation on the surface of the yarns [8]. Therefore, the conductive cotton yarns prepared by the sizing process can improve the penetration of conductive fillers into the cotton yarns by
Table 2 Structural parameters of cotton yarn after sizing

\begin{tabular}{lllllllll}
\hline Counts & Tex & $D \mathrm{~mm}$ & $P \mathrm{~g} / \mathrm{cm}^{3}$ & Twist factor & $I_{\mathrm{c}} \mathrm{mm}$ & $\Phi \%$ & Fiber mass $(\mathrm{g})$ & Fiber number/m \\
\hline $14^{\mathrm{s}}$ & 42.96 & 0.246 & 0.904 & 327.50 & 1.36 & 0.579 & $9.92 \times 10^{-6}$ & 4330 \\
$7^{\mathrm{s}}$ & 82.05 & 0.358 & 0.816 & 226.50 & 1.75 & 0.523 & $14.48 \times 10^{-6}$ & 5664 \\
Remark & 1. The length and diameter of fiber: $14^{\mathrm{s}}: 31 \mathrm{~mm}, 16 \mu \mathrm{m} ; 7^{\mathrm{s}}: 29 \mathrm{~mm}, 20 \mu \mathrm{m}$ \\
& \multicolumn{6}{l}{ 2. No fiber number changes before and after sizing 3. The density of fiber is $1.56 \mathrm{~g} / \mathrm{cm}^{3}$} \\
\hline
\end{tabular}


Table 3 Calculated and measured resistance of yarn with different lengths

\begin{tabular}{lllllll}
\hline Length of yarn $(\mathrm{mm})$ & $25(<\mathrm{If})$ & 50 & 100 & 200 & 400 \\
\hline $14^{\mathrm{s}}$ & $\mathrm{Z}$ & 1 & 2 & 3 & 6 & 13 \\
& $N$ & 128 & 256 & 384 & 768 & 1664 \\
& $n$ & 56 & $56 \times 2$ & $56 \times 3$ & $56 \times 6$ & $56 \times 13$ \\
& $n p$ & 38 & $38 \times 2$ & $38 \times 3$ & $38 \times 6$ & $38 \times 13$ \\
& $n p^{2}$ & 0 & $26 \times 2$ & $26 \times 3$ & $26 \times 6$ & $26 \times 13$ \\
Calculated & $R_{\mathrm{f} 1}(\mathrm{M} \Omega)$ & - & 3.86 & 3.86 & 3.86 & 3.86 \\
$7^{5}$ & $R_{\mathrm{f} 2}(\mathrm{M} \Omega)$ & - & 0.169 & 0.169 & 0.169 & 0.169 \\
& $R_{\mathrm{fL}}(\mathrm{M} \Omega)$ & 3.11 & - & - & - & - \\
& $\mathrm{Ry}(\mathrm{M} \Omega)$ & 0.082 & 0.216 & 0.324 & 0.648 & 1.404 \\
& $\mathrm{Ry}(\mathrm{M} \Omega)^{*}$ & 0.073 & 0.158 & 0.309 & 0.626 & 1.317 \\
& $Z$ & 1 & 2 & 4 & 7 & 14 \\
& $N$ & 154 & 308 & 616 & 1078 & 2156 \\
& $n$ & 67 & $67 \times 2$ & $67 \times 4$ & $67 \times 7$ & $67 \times 14$ \\
& $n$ & 38 & $38 \times 2$ & $38 \times 4$ & $38 \times 7$ & $38 \times 14$ \\
& $n p$ & - & $21 \times 2$ & $21 \times 4$ & $21 \times 7$ & $21 \times 14$ \\
& $n p^{2}$ & - & 2.31 & 2.31 & 2.31 & 2.31 \\
& $R_{\mathrm{f} 1}(\mathrm{M} \Omega)$ & - & 0.14 & 0.14 & 0.14 & 0.14
\end{tabular}

dipping and sizing process and form a conductive coating film on the surface of the yarns by hot wind drying. As a result, the expected better conductivity and stability of cotton yarns can be obtained (Scheme 1). So it is reasonable to analyze the conductive properties and mechanism of the cotton yarn prepared by sizing from the conductive mechanism of the fibers.

\subsection{Conductive mechanism of cotton fiber}

Due to the difference in fiber-to-fiber conductivity in staple spun yarn, it is difficult to define the conductivity of a single fiber. By the analysis of the electrical conductivity of cellulose/graphene composites prepared by hot pressing and impregnation similar to the sizing, the process can be approximately expressed the electrical conductivity of a single fiber. According to our previous study, the bridging effect of amphiphilic graphene dispersants can improve the interaction between cellulose and graphene molecules (Support Information S5), what needs to be further discussed is the effect of GnPs content on the electrical conductivity of the composite. The electrical conductivity of cellulose/GnPs composite containing insulating matrix (cellulose) and conducting inclusions (GnPs) has been well described by the percolation theory in the vicinity of the insulator-conductor transition [19]. Below a critical

Scheme 1 Schematic diagram of conductive cotton yarn prepared by sizing

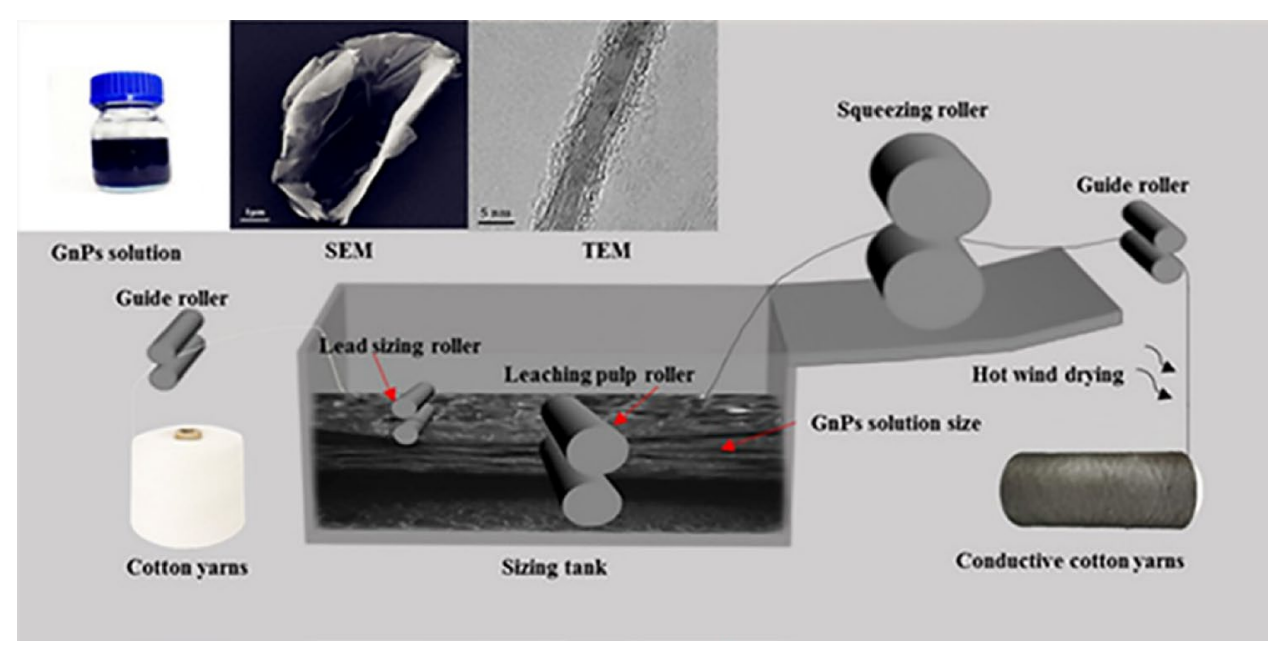


volume fraction of conducting inclusions (fc) GnPs is individually isolated in the insulating matrix, as fc increases, the connection probability of GnPs also increases until a conductive network construction appears corresponding to a sharp change in the conductivity of the composite from the insulator to a conductor. The $\mathrm{fc}$ is the so-called permeation threshold, and the electrical conductivity of composite can be expressed as [20]:

$\sigma_{\mathrm{c}}=\sigma_{\mathrm{e}}\left(f-f_{\mathrm{c}}\right)^{s}$

where $\sigma_{\mathrm{c}}$ is conductivity of composite, $\sigma_{\mathrm{e}}$ is the effective interface conductivity between GnPs and cellulose, $s$ is the critical exponents.

According to the conductivity value of CGP with different GnPs contents (Table 1), it can be found that the good exponential relationship between the conductivity of the CGP and the volume fraction of GnPs, which proves the rationality of the permeation theory to explain the conductive mechanism of cellulose/GnPs composite (Fig. 1a). However, it is a challenge for how to determine the conductivity of the composite material corresponding to the critical volume fraction result from the exponential fitting curve is a continuous function without inflection points. Celzard et al. [3] supposed the equation of the critical volume fraction and the aspect ratio (a) of the conductive filler as:

$1-\exp \left(-\frac{3.6}{\pi} \alpha\right)<f_{\mathrm{c}}<1-\exp \left(-\frac{5.6}{\pi} \alpha\right)$
If the aspect ratio of GnPs in CGP can be reckoned as 0.05 , the value of $5.38 \%<f_{c}<8.62 \%$ can be calculated (Support Information S6). Here, a simple method was proposed to determine the critical volume fraction, as seen in Fig. 1b. The relationship graph between the GnPs stacking density and the electrical conductivity that there are two linearly fitting segments with different slopes and the intersection point corresponds to the electrical conductivity at the critical volume fraction $\left(f_{c} \approx 5.20 \%\right)$, which is nearly consistent with the calculated value from Eq. (2). So far it can be assumed that when the mass fraction of GnPs is more than $2.0 \mathrm{wt} \%$, the volume fraction is greater than $5.20 \%$, the electrical conductivity of the CGP is more than $0.4 \mathrm{~S} / \mathrm{cm}$, the conductivity of cotton fibers expected to be more than $0.4 \mathrm{~S} / \mathrm{cm}$ using a similar sizing process.

\subsection{Conductive mechanism of cotton yarn}

As seen on the SEM image of cotton yarn after sizing, GnPs adhere to the surface of the yarn and penetrates the fiber gaps inside the yarn (Fig. 2a) and the GnPs coated on the surface of the fiber are also clearly visible on the highmagnification SEM image (Fig. 2b), which demonstrates that GnPs can be combined with cotton fiber by sizing.

Due to the structural characteristics of the staple spun yarns, it can be considered that two conditions are required to achieve the conductivity of the cotton yarns. (1) The fibers are conductive. (2) These conductive fibers are connected. The mechanism of fiber conductivity was explained before, and then the connection state of the
Fig. 1 a Volume fraction of GnPs versus resistance. $\mathbf{b}$ Packing density of GnPs versus resistance
Fig. 2 a Side-view SEM image of conductive cotton yarn, $\mathbf{b}$ high-magnification image
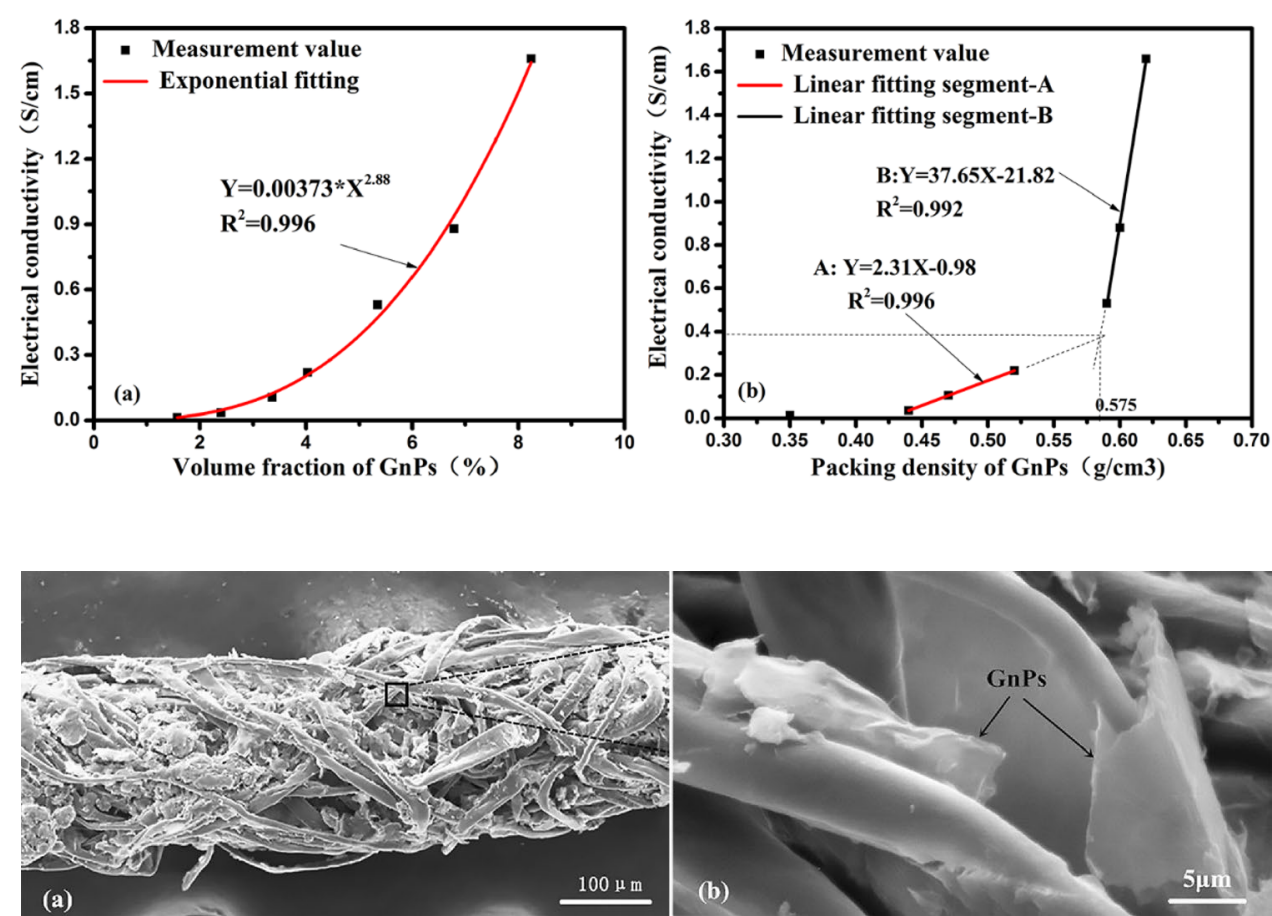
fibers in the yarn can be discussed from the perspective of probability. From the axial distribution of fibers in the yarns, Hearle et al. [11] assumed so-called slip length $\left(I_{c}\right)$ early in 1969 that the fiber in the yarn slips along the axial direction when an external force is applied; as a result, the number of fibers of any cross section in the yarn is different. It can be assumed accordingly that the yarn is alternately divided into the main tensile zone $\left(I_{\mathrm{p}}\right)$ and the slipping zone $\left(I_{c}\right)$ along the axial direction, and the conductive channel of the yarn is composed of the conductive fibers that have been connected in the main tensile zone pass through the slipping zone and then being connected to the conductive fibers in the next main tensile zone (Fig. 3). Here, $I_{\mathrm{p}}+I_{\mathrm{c}}=I_{\mathrm{f}}, I_{\mathrm{f}}$ is the length of fiber, $I_{c}$ can be calculated by equation of Jiang et al. (Support Information S7). From the radial distribution of fibers in the yarns, Schwarz [21] proposed a fiber distribution model for dense stacking and calculated the filling coefficients as $0.7-0.8$. Hamilton [9] et al. demonstrated that most of the fiber filling coefficients in actual yarns were less than 0.7 (Fig. 4). It can be assumed that when the filling coefficients are beyond 0.7, all fibers are connected (the connection probability $p$ is 1 ), and the connection probability density function is proportional to the filling coefficients which can be expressed as:

$f(\varphi)=k \varphi \int_{0}^{0.7} f(\varphi) \mathrm{d} \varphi=1$

$p=\int_{0}^{\varphi} f(\varphi) \mathrm{d} \varphi=2.04 \varphi^{2}$

$f(\varphi)$ is connection probability density function, $k$ is the proportionality coefficients calculated as 2.04.

As above mentioned when the mass fraction of $\mathrm{GnPs}$ exceeds $2.0 \mathrm{wt} \%$, the average conductivity of the fiber is above $0.4 \mathrm{~S} / \mathrm{cm}$. Since the penetration of GnPs solution in the yarn presents a radial gradient distribution, fibers in the outer layer exhibit higher conductivity than in the
Fig. 3 Schematic diagram of fibers axis distribution and zone connection
Fig. 4 Schematic diagram of radial fibers distribution: a tightly packing model, $\mathbf{b}$ actual model

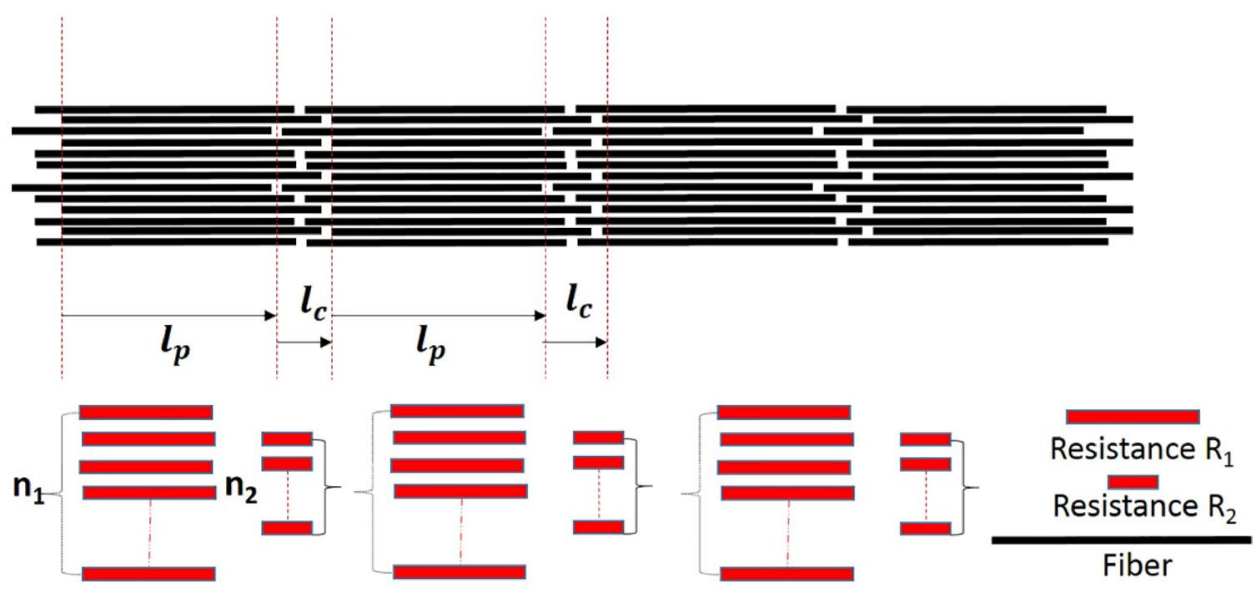

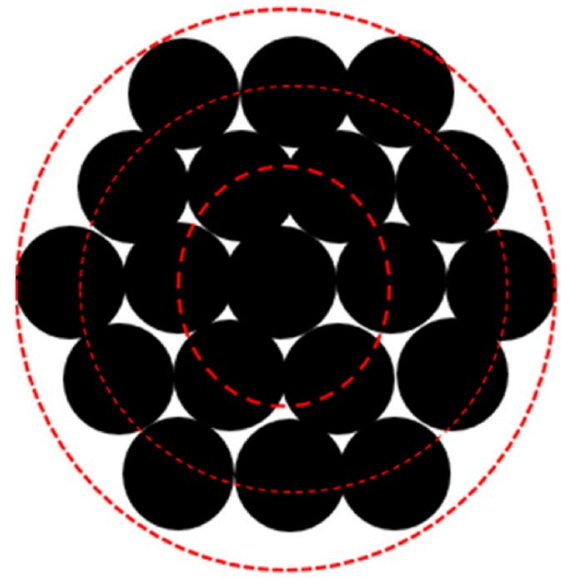

(a)

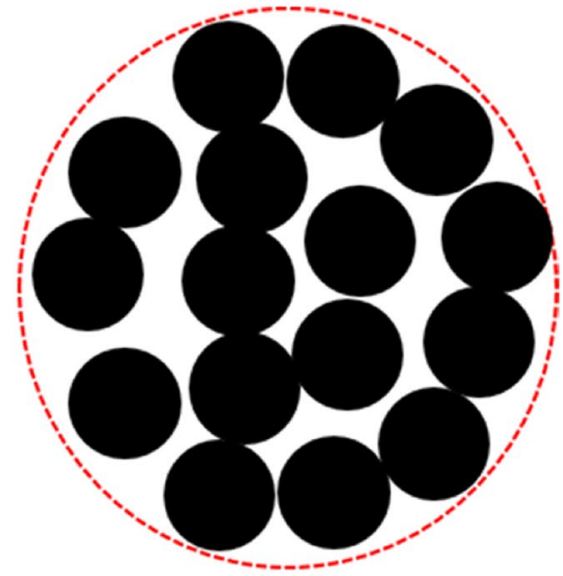

(b) 
inner layer. It can be assumed that the number of conductivity fibers is proportional to the yarn radius as seen in Fig. 5. Then, the conductive fibers $(n)$ and a total number of fibers $(N)$ in a certain length of yarn can be expressed as:

$n=k r \quad N=\int_{0}^{r_{1}} n 2 \pi \mathrm{d} r=\int_{0}^{r_{1}} k r 2 \pi \mathrm{d} r=\frac{2 \pi k r_{1}^{3}}{3}$

$K$ is the proportionality coefficients calculated as $\frac{3 N}{2 \pi r_{1}^{3}}$.

The position of the conductive fibers with a weighted average radius is:

$r_{2}=\frac{r \int_{0}^{r_{1}} k r 2 \pi r d r}{N}=\frac{3}{4} r_{1}$

The conductivity of the fibers within the range of $r_{2}$ to $r_{1}$ exceeds the average conductivity corresponding to the critical threshold $(0.4 \mathrm{~S} / \mathrm{cm})$, and these fibers are considered to be effective conductive fibers ( $n$ ). However, the fibers in radial less than $\mathrm{r}_{2}$ do not reach the critical threshold and are considered to be noneffective conductive fibers. Assuming that the fibers distributed in the yarn cross section are uniform, the relationship between the number and the occupied area of fibers can be expressed as:

$$
\begin{aligned}
n & =\frac{\pi r_{1}^{2}-\pi r_{2}^{2}}{\pi r_{1}^{2}} N \\
& =\frac{7 N}{16}
\end{aligned}
$$

According to the definition of the binomial distribution, when the connection probability of each fiber is $p$, the probability of the event $X$ (with the connection fiber number $i$ in a total number of effective conductive fibers $n$ ) and its expected value of event $X$ can be expressed as:

$p\left(X_{i}\right)=\left(\begin{array}{c}n \\ i\end{array}\right) p^{i}(1-p)^{n-i}$
$E\left(X_{i}\right)=n p^{2}$

Based on Eq. (9), it can be assumed that the maximum possible number of fibers connected in the main tensile zone is $n \times p$.

The fibers in the slipping zone are connected through the fibers already connected in the main tensile zone, and similarly, according to the definition of the binomial distribution, the connection probability of fiber number of $i$ and its expectation can be expressed as:

$p\left(x_{i}\right)=\left(\begin{array}{c}n p \\ i\end{array}\right) p^{i}(1-p)^{n p-i} \quad E\left(X_{i}\right)=n P^{2}$

For a yarn of any length $\left(L>I_{\mathrm{f}}\right)$, it can be regarded as a long segment composed of several main tensile areas and slipping zone, and each main tensile zone followed by a slipping zone. The fiber resistance in each zone is in parallel, while the fiber resistance between the zones is in series (Fig. 3). The resistance of yarn with given length $L$ can be expressed as:

$R_{y}=\frac{R_{\mathrm{f} 1}}{n p}+z \frac{R_{\mathrm{f} 2}}{n p^{2}} \quad L>I_{\mathrm{f}}$

$R_{y}=\frac{R_{\mathrm{fL}}}{n p} \quad L<I_{\mathrm{f}}$

$z=\operatorname{INT}\left(\frac{L}{l_{\mathrm{f}}}+0.5\right)$

$R_{\mathrm{f} 1}=\frac{4}{\sigma_{\mathrm{c}}} \frac{I_{\mathrm{p}}}{\pi d^{2}} \quad R_{\mathrm{f} 2}=\frac{4}{\sigma_{\mathrm{c}}} \frac{I_{\mathrm{c}}}{\pi d^{2}} \quad R_{\mathrm{fL}}=\frac{4}{\sigma_{\mathrm{c}}} \frac{L}{\pi d^{2}}$

$R_{\mathrm{f} 1}, R_{\mathrm{f} 2}, R_{\mathrm{fL}}$, is the resistance of fiber with the length of $I_{p}, I_{c}, L, z$ is the number of zones calculated by integer after rounding.
Fig. 5 Radial gradient distribution the conductivity of cotton yarn. a Cross-section SEM image. $\mathbf{b}$ Schematic diagram of radial distribution of conductive fibers

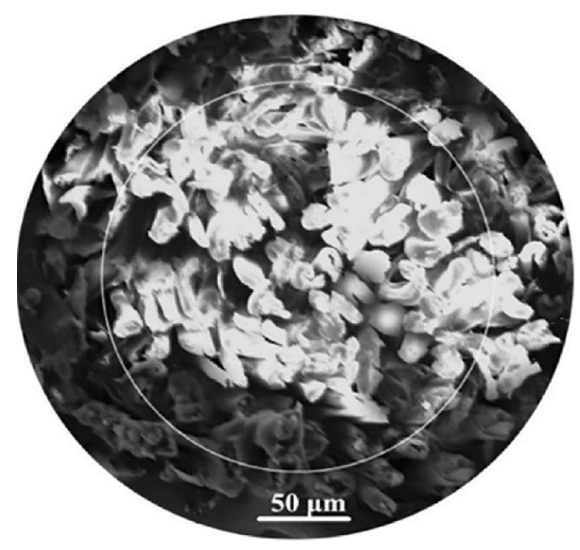

(a)

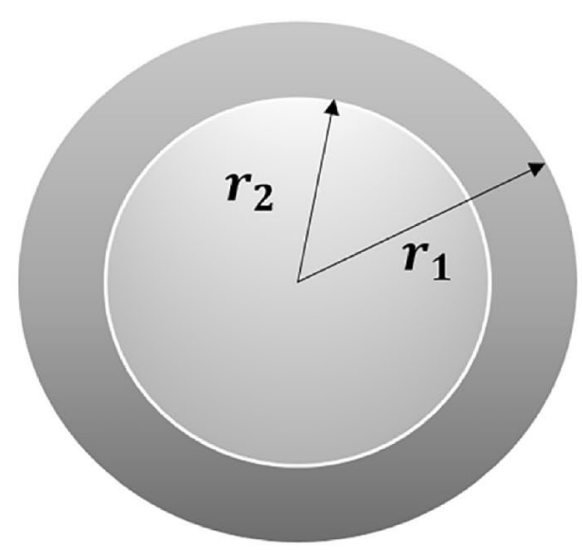

(b) 


\subsection{Experimental verification}

The calculated resistance of yarns with a different lengths according to Eqs. (11), (12) is listed in Table 3, and the resistance was also measured for comparison. As seen from the curve of resistance vs yarn's length (Fig. 6), it can be found that the resistance increases with the measured length of yarn and conforms to the resistance calculation formula $R_{y}=\frac{4 L}{\sigma_{y} \pi D^{2}}$, which indicates that the conductivity of yarn $\left(\sigma_{y}\right)$ can be derived by the slope of the linear fitting $(0.65 \mathrm{~S} / \mathrm{cm}$ for the yarn of $14 \mathrm{~s}$ and $0.45 \mathrm{~S} / \mathrm{cm}$ for the yarn of $7^{\mathrm{s}}$ ). More noticeable is that the ratio of the yarn conductivity is very close to the square of the connection probability of the yarn, which further confirms that the yarn conductivity is related to the fiber connection probability [Eq. (15)]. The twist inserted, quality of the yarn, and filling coefficient of the fibers affect the conductivity. With the probability of connection of fibers increase the conductivity of yarns is higher (e. g. the conductivity of yarn $14 \mathrm{~s}$ is higher than $7 \mathrm{~s}$ ). It should be pointed out that because of pretension applied on the yarn during the measurement the measured resistance value is less than the calculated.

$$
\frac{\sigma_{y\left(7^{5}\right)}}{\sigma_{y\left(14^{s}\right)}}=\frac{0.44}{0.65}=0.676 \quad\left(\frac{p_{\left(7^{5}\right)}}{p_{\left(14^{5}\right)}}\right)^{2}=\left(\frac{0.56}{0.68}\right)^{2}=0.678
$$

In addition, the conductivity of the cotton yarn prepared by the sizing process can be reduced to the $\mathrm{k} \Omega$ level; this result is better than other conductive cotton yarns coated with graphene as mentioned above [14, 18] and even is equivalent to that of the cotton fabrics also by coating graphene. Chatterjee et al. [4] prepared

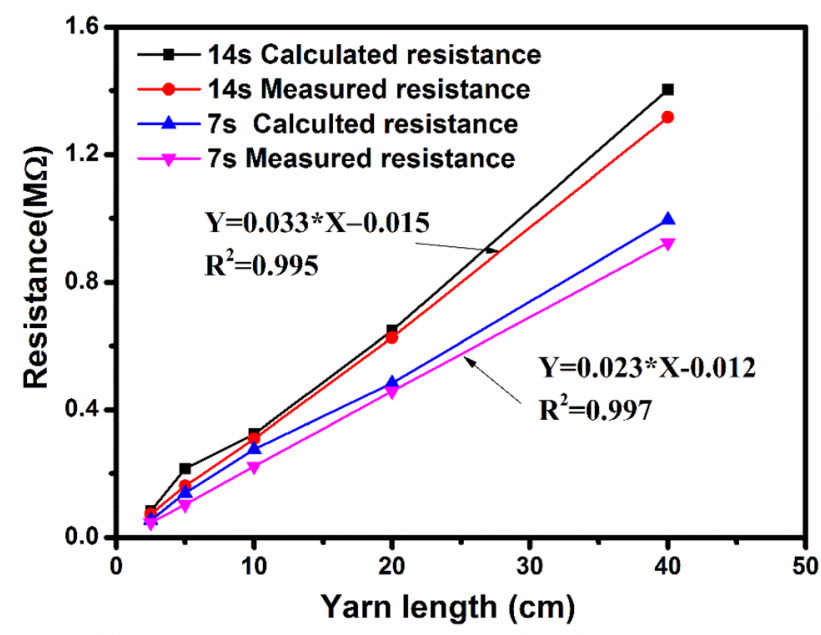

Fig. 6 Comparison of cotton yarn resistance the measured and calculated value conductive cotton fabric by RGO dip coating with resistivity of $190 \mathrm{k} \Omega / \mathrm{sq}$. He et al. [10] prepared the conductive cotton fabric with resistivity of $76.3 \mathrm{k} \Omega / \mathrm{sq}$ using the same method. In general, the fabrics form more conductive channels due to the interweaving relationship of the yarns, so its resistance is lower than that of the yarns. The above results further illustrate the feasibility of preparing conductive cotton yarn by sizing process.

\subsection{The influence of temperature and humidity on cotton yarn resistance}

The influence of temperature and humidity on the electrical conductivity of cotton yarns has to consider, due to the excellent thermal conductivity of graphene $(5300 \mathrm{~W} / \mathrm{mK})$ and the good hygroscopicity of cotton fibers (7-8wt\%) [5, 24]. Wang et al. [25] demonstrated the negative temperature coefficient (NTC) feature of graphene incorporated in hydrophilic sodium alginate substrate which indicated that the resistance of the graphene-based materials decreases as the temperature rises. Cusick and Hearle [6] proved the inverse relationship between resistance and moisture content of cotton fiber early in 1955. As seen in Fig. 7, the resistance of different yarns ( $14 \mathrm{~s}, 7 \mathrm{~s}$ ) exhibits a trend to monotonous decline with increasing humidity and temperature. The yarns with the tighter the structure, the better the linearity of the resistance decline trend. The influence of temperature and humidity for resistance can be derived by the slope of the linear fitting line and then the influence of temperature and humidity on the initial resistance can be corrected.

\subsection{The influence of washing fastness on cotton yarn resistance}

As seen in Fig. 8, it can be found from the resistance change curve of the yarn immersed in water for different times that at the beginning of the period the resistance will increase rapidly, which may be due to the detachment of the surface GnPs into the water in the initial period; then, the resistance will stabilize at a certain value which further indicates the better interaction between the cellulose and GnPs. The stable resistance value of the yarns is relative with time, $7^{5}$ yarns (20 min) is less than that of the $14^{\mathrm{S}}$ yarns with a tighter structure (30 min). Although the stable resistance has nearly threefold after immersed in water for $20 \mathrm{~min}$, the washing fastness of the yarn resistance can be maintained by surface protection coating treatment similar to laminated fabrics [28]. 
Fig. 7 Influence of temperature and humidity on yarn resistance: a cotton yarn resistance under different humidity, b cotton yarn resistance under different temperature
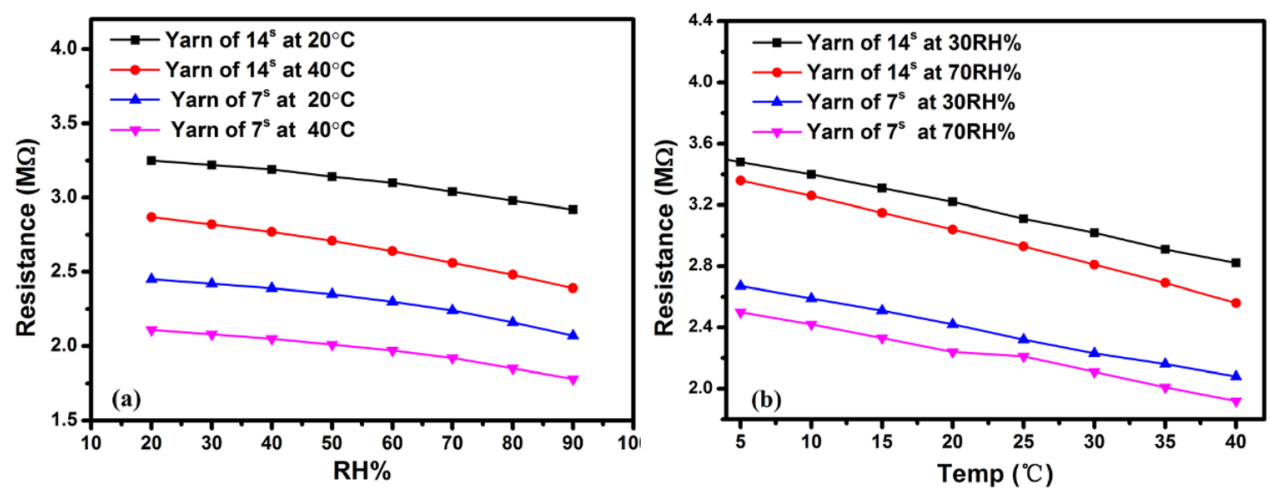

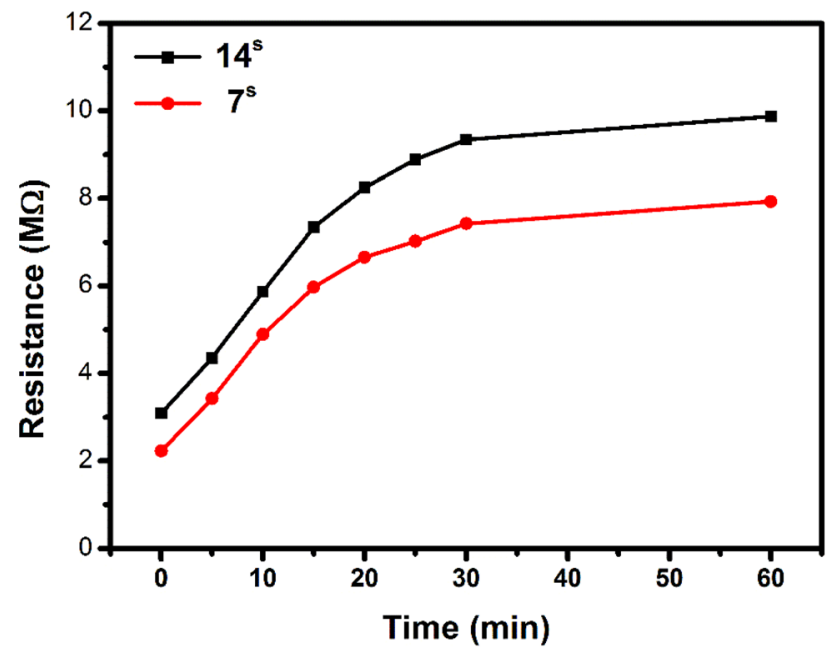

Fig. 8 Washing fastness of the yarn resistance at different immerged time in water

\section{Conclusion}

In summary, the electron conductive cotton yarns with a conductivity of $0.65 \mathrm{~S} / \mathrm{cm}$ can be prepared by a simple sizing process. By analysis of the conductive mechanism of fibers and yarns, the resistance calculation formula of staple spun yarn can be derived. Meanwhile, it is concluded that the performance of conductive cotton yarns can be improved by increasing the fineness of fibers, the conductive properties of fibers, and the filling coefficient of fibers in yarns, and the influence of temperature and humidity on yarn's conductivity can also be corrected initially. This work fully demonstrates the idea of preparing conductive cotton yarn by sizing for the first time, and we expect the sizing process will be widely used in the functional treatment of cotton yarns.

Acknowledgements The work was financially supported by the Public Technology Research Plan of Zhejiang Province (LGF18E030003),
National Natural Science Foundation of China (51672251), and 521 Talent Project of Zhejiang Sci-Tech University.

Declaration

Conflict of interest The authors declare that they have no conflict of interest.

Open Access This article is licensed under a Creative Commons Attribution 4.0 International License, which permits use, sharing, adaptation, distribution and reproduction in any medium or format, as long as you give appropriate credit to the original author(s) and the source, provide a link to the Creative Commons licence, and indicate if changes were made. The images or other third party material in this article are included in the article's Creative Commons licence, unless indicated otherwise in a credit line to the material. If material is not included in the article's Creative Commons licence and your intended use is not permitted by statutory regulation or exceeds the permitted use, you will need to obtain permission directly from the copyright holder. To view a copy of this licence, visit http://creativecommons. org/licenses/by/4.0/.

\section{References}

1. Asghar A, Ahmad MR, Yahya MF (2016) Effects of metal filament's alignment on tensile and electrical properties of conductive hybrid cover yarns. Fash\&Text 3(1):1-12. https://doi. org/10.1186/s40691-015-0055-4

2. Cai F, Chen T, Peng H (2012) All carbon nanotube fiber electrode-based dye-sensitized photovoltaic wire. J Mater Chem 22(30):14856-14860. https://doi.org/10.1039/C2JM32256K

3. Celzard A, McRae E, Deleuze C, Dufort M, Furdin G, Marêché JF (1996) Critical concentration in percolating systems containing a high-aspect-ratio filler. Phy Rev B 53(10):6209-6214. https://doi.org/10.1103/PhysRevB.53.6209

4. Chatterjee A, Nivas Kumar M, Maity S (2017) Influence of graphene oxide concentration and dipping cycles on electrical conductivity of coated cotton textiles. J Text Inst 108:19101916. https://doi.org/10.1080/00405000.2017.1300209

5. Chen S, Wu Q, Mishra C, Kang J, Zhang H, Cho K, Cai W, Balandin A, Ruoff R (2012) Thermal conductivity of isotopically modified graphene. Nat Mater 11:203-207. https://doi.org/10. 1038/nmat3207

6. Cusick GE, Hearle JWS (1955) The electrical resistance of synthetic and cellulose acetate Fibres. J Text I 46:T699-T711. https://doi.org/10.1080/19447027.1955.10750363

7. Das BR, Ishtiaque SM, Rengasamy RS (2010) Static failure mechanism of staple yarns: a critical review. J Text App 
Technol Manag 1(1):50-58. https://doi.org/10.3923/ajt.2011. 50.58

8. Gandhi KL (2012) Yarn preparation for weaving: sizing. Woven Text. https://doi.org/10.1533/9780857095589.1.85

9. Hamilton JB (1959) 40-A direct method for measuring yarn diameters and bulk densities under conditions of thread flattening. J Text Inst Trans 50:T655-T672. https://doi.org/10. 1080/19447025908659944

10. He S, Xin B, Chen Z, Liu Y (2018) Functionalization of cotton by reduced graphene oxide for improved electrical conductivity. Text Res J 89:1038-1050. https://doi.org/10.1177/0040517518 760757

11. Hearle JWS, Grosberg P, Backer S (1969) Structural mechanics of fibers, yarns, and fabrics. Structural Mechanics of Fibers Yarns \& Fabrics

12. Huang CT, Shen $C L$, Tang CF, Chang SH (2008) A wearable yarnbased piezo-resistive sensor. Sens Actuat A Phys 141:396-403. https://doi.org/10.1016/j.sna.2007.10.069

13. Huang Y, Ip WS, Lau YY, Sun J, Zeng J, Yeung N, Ng WS, Li H, Pei Z, Xue Q, Wang Y, Yu J, Hu H (2017) Weavable, conductive yarn-based $\mathrm{NiCo} / / \mathrm{Zn}$ textile battery with high energy density and rate capability. ACS Nano 11:8953-8961. https://doi.org/ 10.1021/acsnano.7b03322

14. Kim E, Arul NS, Han JI (2016) Electrical properties of conductive cotton yarn coated with eosin $\mathrm{Y}$ functionalized reduced graphene oxide. J Nanosci Nanotechnol 16:6061-6067. https:// doi.org/10.1166/jnn.2016.10856

15. Kou L et al (2014) Coaxial wet-spun yarn supercapacitors for high-energy density and safe wearable electronics. Nat Commun 5:3754-3762. https://doi.org/10.1038/ncomms4754

16. Lee J, Llerena Zambrano B, Woo J, Yoon K, Lee T (2020) Recent advances in 1D stretchable electrodes and devices for textile and wearable electronics: materials, fabrications, and applications. Adv Mater 32(5):1-28. https://doi.org/10.1002/adma. 201902532

17. Liu Y, Liu L, Li Z, Zhao Y, Yao J (2019) Green and facile fabrication of smart cellulose composites assembled by graphene nanoplates for dual sensing. Cellulose 26:9255-9268. https://doi.org/ 10.1007/s10570-019-02735-z

18. Ma H, Wu W, Cao J, Yue B, Zhang H (2017) Network structure and electromechanical properties of viscose-graphene conductive yarn assembles. Carbon 114:731-739. https://doi.org/10.1016/j. carbon.2016.12.063

19. Mutlay I, Tudoran LB (2014) Percolation behavior of electrically conductive graphene nanoplatelets/polymer nanocomposites: theory and experiment. Fuller Nanotubes Carbon Nanostruct. 22:413-433. https://doi.org/10.1080/1536383x.2012.684186

20. Nan CW, Shen Y, Ma J (2010) Physical properties of composites near percolation. Annu Rev Mater Res 40:131-151. https://doi. org/10.1146/annurev-matsci-070909-104529

21. Schwarz ER (1950) Twist structure of plied yarns. Text Res J 20:175-179. https://doi.org/10.1177/004051755002000305

22. Sim CYD, Tseng CW, Leu HJ (2012) Embroidered wearable antenna for ultrawideband applications. Microw Opt Technol Lett 54:2597-2600. https://doi.org/10.1002/mop.27133

23. Sluis OVD, Hsu YY, Timmermans PHM, Gonzalez M, Hoefnagels JPM (2011) Stretching-induced interconnect delamination in stretchable electronic circuits. J Phys Appl Phys 21:467-478. https://doi.org/10.1111/1467-8365.00125

24. Su Y, Li R, Yang J, Xiang C, Song G, Li J (2019) Influence of transport properties of laminated membrane-fabric on thermal protective performance against steam hazard. Fiber Polym 20:2433-2442. https://doi.org/10.1007/s12221-019-1155-3

25. Walker AC (1933) Effect of atmospheric humidity and temperature on the relation between moisture content and electrical conductivity of cotton*. Bell Syst Technol J 24:T145-T160. https://doi.org/10.1080/19447023308661584

26. Wang F, Jiang J, Sun F, Sun L, Wang T, Liu Y, Li M (2019) Flexible wearable graphene/alginate composite non-woven fabric temperature sensor with high sensitivity and anti-interference. Cellulose 27:2369-2380. https://doi.org/10.1007/s1057 0019-02951-7

27. Xu Z et al (2016) Ultrastiff and strong graphene fibers via fullscale synergetic defect engineering. Adv Mater 28:6449-6456. https://doi.org/10.1002/adma.201506426

28. Yongwoo J, Sung MK, Keon JK, Hyeon JS, Seon JK (2019) Selfpowered coiled carbon-nanotube yarn sensor for gastric electronics. ACS Sens 4:2893-2899. https://doi.org/10.1021/acsse nsors.9b01180

29. Yu X et al (2017) A coaxial triboelectric nanogenerator fiber for energy harvesting and sensing under deformation. J Mater Chem A 5:6032-6037. https://doi.org/10.1039/c7ta00248c

30. Zeng W, Shu L, Li Q, Chen S, Wang F, Tao XM (2014) Fiber-based wearable electronics: a review of materials, fabrication, devices, and applications. Adv Mater 26(31):5310-5322. https://doi.org/ 10.1002/adma.201400633

Publisher's Note Springer Nature remains neutral with regard to jurisdictional claims in published maps and institutional affiliations. 